\title{
Effect of Heat-Producing Needling Technique on the Local Skin Temperature: Clinical Dataset
}

\author{
Zehuan Liao@ and Yan Zhao* \\ School of Biological Sciences, Nanyang Technological University, 60 Nanyang Drive, Singapore 637551, Singapore; \\ liao0058@e.ntu.edu.sg \\ * Correspondence: zhaoyan@ntu.edu.sg; Tel.: +65-6514-1901
}

Received: 28 October 2018; Accepted: 11 December 2018; Published: 12 December 2018

check for updates

\begin{abstract}
The heat-producing needling technique is a special compound manipulating procedure on the acupuncture needle which has been recorded to produce a warm sensation in the body in ancient TCM literature. This randomized, subject-blinded clinical study was performed to examine the effect of heat-producing acupuncture treatment on the ST36 local skin temperature. A total of 30 healthy participants received four successive sessions of heat-producing acupuncture treatment, non-acupoint heat-producing acupuncture treatment, normal stable acupuncture treatment, and non-invasive sham acupuncture treatment at the ST36 acupoint in a random sequence. Within each session, the local ST36 skin temperature and basal body temperature of each participant were measured at 1 min before needle insertion, just after needle insertion and manipulation (if any), 5 min after needle insertion with needle removal immediately after temperature taking, and 5 min after needle removal. Furthermore, the participants were also required to declare their needling and heat sensation felt during the acupuncture needling treatment period using a visual analogue scale from 1 to 10 immediately after each treatment session. This data descriptor presents all the clinical data obtained in the above mentioned study.
\end{abstract}

Dataset: Supplementary data to this article.

\section{Dataset License: CC-BY-4.0}

Keywords: heat-producing needling; acupuncture; Shao Shan Huo; setting fire on the mountain; local skin temperature; body temperature; traditional Chinese medicine; visual analogue scale

\section{Summary}

Since ancient times, according to the patients' subjective feelings and reports, acupuncture practitioners have realized that some specific acupuncture techniques may be able to manipulate the local skin or body temperature [1]. The heat-producing needling technique (Shao Shan Huo, literally burning-mountain-fire in Chinese), also known as "setting fire on the mountain" in scientific literature, is a special compound manipulating procedure on the acupuncture needle which has been recorded to produce a warm sensation in the body in ancient TCM literature. In the Yellow Emperor's Classic of Medicine (Huang Di Nei Jing), it is stated that acupuncture is "puncturing for deficiency can bring about excess which produces heat beneath the needle". The Ode to the Golden Needle (Jin Zhen Fu) reports that the heat-producing needling technique "treats obstinate numbness and cold pain". The Great Compendium of Acupuncture and Moxibustion (Zhen Jiu Da Cheng) also states that the heat-producing needling technique "can dissipate cold" [2]. In short, the heat-producing needling technique is basically a combination of lifting and thrusting, as well as twisting and rotating manipulations of the acupuncture needle during needle insertion. 
Historically, this acupuncture technique has been used to treat many common conditions, such as cold limbs, abdominal pain, and diarrhea.

On the biochemical level, previous studies have demonstrated that increased nitric oxide (NO) synthase activity has been found in traditionally identified meridians and acupoints [3]. Therefore, NO synthase expression is higher in meridian skin regions and acupoints compared to the other areas. In a sham-controlled study, NO concentration was significantly increased in the plasma from the acupunctured (needles at Li4, P6, P8 L6, and H5 acupoints) arm at 60 min after acupuncture [4]. Furthermore, in another study, it is also demonstrated that blood perfusion at ST36 acupoint was significantly increased after the lifting-thrusting manipulation of acupuncture [5]. NO is a key regulator of local blood circulation and hence, the change in local blood circulation may be a plausible explanation for the change in local skin temperature. However, more clinical studies are required to prove this hypothesis.

This clinical study aims to examine the influence of heat-producing needling acupuncture treatment on the local skin temperature of ST36 acupoint.

\section{Data Description}

The supplementary MS Excel file contains all anonymous clinical data obtained in the study of "Effect of Heat-Producing Acupuncture Technique on the Local Skin Temperature". MS Excel Sheet 1 contains the 30 volunteers' basic biodata (gender, race, year of birth, and experience with acupuncture). MS Excel Sheet $2-5$ contains the raw data obtained in heat-producing acupuncture, non-acupoint heat-producing acupuncture, normal stable acupuncture, and non-invasive sham acupuncture treatment groups, respectively. In each MS Excel Sheet of 2-5, basal body temperature and local skin temperature at $1 \mathrm{~min}$ before the needle insertion (T1B), just after needle insertion (T0), $5 \mathrm{~min}$ after needle insertion with immediate needle removal after temperature taking (T5), and 5 min after needle removal (T5A) are recorded. All recorded temperatures have the unit of degree Celsius $\left({ }^{\circ} \mathrm{C}\right)$. Visual Analogue Scale (VAS) scores for needling sensation and heat sensation are also recorded in MS Excel Sheet of 2-5. List of abbreviations used in the MS Excel Sheets are presented in Table A1 of the Appendix A.

\section{Methods}

This is a subject-blinded, sham-controlled single center clinical study conducted in the year of 2017 at the School of Biological Sciences, Nanyang Technological University.

\subsection{Ethics Declaration}

This clinical study was conducted in accordance with the Declaration of Helsinki. The experimental procedure was reviewed and endorsed by the Institutional Review Board (IRB) of Nanyang Technological University (IRB Application Code: IRB-2017-06-016). All participants were briefed, gave written informed consent before the study, and were allowed to exit the study freely whenever necessary.

\subsection{Selection of Participants}

Through recruitment in the university compound, 30 healthy Chinese participants with an equal number of males and females and an age range between 19-23 years old inclusive volunteered and completed this study. All recruited participants declared having no existing medical conditions before and during the study. Furthermore, 26 participants declared having received acupuncture as a treatment before the study, but all participants had minimal knowledge of acupuncture.

\subsection{Study Venue and Environment}

All acupuncture treatments and temperature takings were conducted at the School of Biological Sciences, Nanyang Technological University, Singapore. The temperature of the building was maintained at $25^{\circ} \mathrm{C}$ by a central air-conditioning system. 


\subsection{Chosen Acupoint}

Each acupuncture treatment was performed on the traditionally identified ST36 acupoint of every participant. The ST36 acupoint, also known as the Zusanli acupoint, is located four finger widths (reference from the person receiving the treatment) or three cun down from the bottom of the knee cap, along the outer boundary of the shin bone of the participant. The ST36 acupoint was chosen for this study due to the following reasons. Firstly, as the ST36 acupoint is located on the lower body, it was relatively easy to blind every participant in this subject-blinded study. Secondly, it was very convenient to perform acupuncture manipulations on the ST36 acupoint as this acupoint was readily reachable (often not covered by clothing) by the acupuncture practitioner. Lastly, the ST36 acupoint was chosen due to safety reasons. The ST36 acupoint has been used in numerous clinical studies and reported complications arising from acupuncture manipulations on this acupoint by licensed acupuncture practitioners are extremely rare.

\subsection{Randomization and Blinding}

Each participant was allocated to undergo four different acupuncture treatment sessions (one heat-producing acupuncture treatment session, one non-acupoint heat-producing acupuncture treatment session, one normal stable acupuncture treatment session, and one non-invasive sham acupuncture treatment session) in different sequences following the principle of simple randomization. The four different acupuncture treatment sessions led to 24 different permutations of acupuncture treatment sessions. Lots with numbers 1-24 were placed in a box and a number corresponding to each participant was drawn with lot replacement. Participants were allocated the acupuncture treatment session sequence based on the number drawn. As a safety precaution, successive treatment sessions were performed at least two days apart.

The participants were blinded in this study and hence, they were not informed of their exact acupuncture treatment session sequences. In order to avoid any discrepancies in the acupuncture manipulations in the same treatment group sessions, all acupuncture treatments were performed by the same certified and experienced acupuncture practitioner. The acupuncture practitioner was only allowed to have minimum verbal interactions with the participants. The temperatures were self-recorded by each participant blinded in this study in order to minimize observer biasness, with the help of a research study assistant for using the temperature taking instruments.

\subsection{Measurement of Temperature}

The participants were asked to come into the room about $10 \mathrm{~min}$ ahead of the schedule to adapt to the environmental temperature. The local skin temperature at ST36 was measured using a Medicare Non-Contact Infrared Thermoscope MC-303. The basal body temperature was measured using a HuBDIC Infrared Ear Thermometer TB-100 Thermo Buddy. Both temperature measuring instruments are currently among the most accurate and precise body and skin temperature measuring instruments in the market. During the study, both thermometers were applied, at $1 \mathrm{~min}$ before the needle insertion (T1B), just after needle insertion (T0), 5 min after needle insertion with immediate needle removal after temperature taking (T5), and $5 \mathrm{~min}$ after needle removal (T5A). Since each participant underwent four treatment sessions, every participant had four series of local skin temperature measurements and four series of basal body temperature measurements.

\subsection{Acupuncture Manipulations}

The participant sat comfortably on a chair and exposed the right lower leg. Acupuncture treatment was then performed on the ST36 acupoint of the participant. As a general workflow, participants rested at the venue for 5-10 mins; were informed; and had their temperature taken at T1B, T0, T5, and T5A. How the acupuncture manipulations were performed in each treatment session was as below. 
Heat-Producing Acupuncture Treatment (HPA): This acupuncture stimulation was done manually using single-use acupuncture needles $(0.30 \times 40 \mathrm{~mm}$, Hanyi Brand). The practitioner inserted the needle on the ST36 acupoint and performed a heat-producing needling technique. After the manipulations, the needle remained at ST36 acupoint stably.

Non-Acupoint Heat-Producing Acupuncture Treatment (NAHPA): This acupuncture stimulation was done manually using single-use acupuncture needles $(0.30 \times 40 \mathrm{~mm}$, Hanyi Brand). The practitioner inserted the needle on the point about $1 \mathrm{~cm}$ below the ST36 acupoint and performed a heat-producing needling technique. After the manipulations, the needle remained in the same place stably.

Normal stable acupuncture (Norm): This acupuncture stimulation was done manually using single-use acupuncture needles $(0.30 \times 40 \mathrm{~mm}$, Hanyi Brand). The practitioner inserted the needle on the ST36 acupoint without any manipulation. The insertion was stopped and the needle remained at ST36 acupoint stably.

Non-invasive sham acupuncture (Sham): This acupuncture stimulation was done manually using non-invasive acupuncture needles specially designed for this experiment. Acupuncture needles $(0.30 \times 40 \mathrm{~mm}$, Hanyi Brand $)$ had their sharp tips removed and attached with a "sticky end". The practitioner "sticked" the needle on ST36 with some pressure and this non-invasive needle remained "sticked" to ST36 acupoint stably (Placebo Treatment).

\subsection{Needling and Heat Sensation}

After every needle insertion and manipulation, volunteers were asked about their perceived needling and heat sensation felt during the acupuncture treatment period (T0-T5). Both the needling and heat sensation were assessed using VAS. For the needling sensation (VAS Sense), a VAS score of 1 refers to "no sensation at all" and a VAS score of 10 refers to "too much sensation". For the heat sensation (VAS Heat), a VAS score of 1 refers to "no heat sensation at all" and a VAS score of 10 refers to "burning hot sensation".

\section{User Notes}

The clinical data on local skin and body temperature at different time periods can be further analyzed to explore if local skin and body temperatures increase significantly in the heat-producing acupuncture treatment group compared to those of non-acupoint heat-producing acupuncture, normal stable acupuncture, and non-invasive sham acupuncture treatment groups. VAS Sense can be compared with the increase in temperature to verify if the temperature increase is due to needling sensation. VAS Heat can be compared with the increase in temperature to verify if VAS is an accurate method to study acupuncture. This dataset can also be used in the meta-analysis of acupuncture treatments.

Supplementary Materials: Supplementary materials can be found at http:/ /www.mdpi.com/2306-5729/3/4/65/s1.

Author Contributions: Conceptualization, Z.L. and Y.Z.; Methodology, Z.L.; Validation, Y.Z.; Formal Analysis, Z.L.; Investigation, Z.L.; Resources, Y.Z.; Data Curation, Z.L.; Writing-Original Draft Preparation, Z.L.; Writing-Review and Editing, Y.Z.; Supervision, Y.Z.; Project Administration, Y.Z.; Funding Acquisition, Y.Z.

Funding: All associated costs in this research were funded by Nanyang Technological University: FYP04.

Conflicts of Interest: The authors declare no conflict of interest.

\section{Appendix A}

Table A1 lists the abbreviations used in the MS Excel Sheets. 
Table A1. List of the abbreviations used in the Supplementary MS Excel File.

\begin{tabular}{cc}
\hline Abbreviations & Full Title \\
\hline HPA & Heat-Producing Acupuncture Treatment \\
NAHPA & Non-Acupoint Heat-Producing Acupuncture Treatment \\
Norm & Normal Stable Acupuncture Treatment \\
Sham & Non-Invasive Sham Acupuncture Treatment \\
Skin T1B & Local Skin Temperature at 1 min before the needle insertion \\
Skin T0 & Local Skin Temperature at just after needle insertion \\
Skin T5 & Local Skin Temperature at 5 min after needle insertion \\
Skin T5A & Local Skin Temperature at 5 min after needle removal \\
Body T1B & Basal Body Temperature at 1 min before the needle insertion \\
Body T0 & Basal Body Temperature at just after needle insertion \\
Body T5 & Basal Body Temperature at 5 min after needle insertion \\
Body T5A & Basal Body Temperature at 5 min after needle removal \\
VAS Sense & Visual Analogue Scale Score for Needling Sensation \\
VAS Heat & Visual Analogue Scale Score for Heat Sensation \\
\hline
\end{tabular}

\section{References}

1. Huang, T.; Huang, X.; Zhang, W.; Jia, S.; Cheng, X.; Litscher, G. The influence of different acupuncture manipulations on the skin temperature of an acupoint. Evid.-Based Complement. Altern. Med. 2013. [CrossRef] [PubMed]

2. Jiang, W.; Liu, W. "Setting Fire on the Mountain" and "Coolness through Penetrating Heaven" Revisited. J. Chin. Med. 2006, 80, 29-33.

3. Ma, S.X. Enhanced nitric oxide concentrations and expression of nitric oxide synthase in acupuncture points/meridians. J. Altern. Complement. Med. 2003, 9, 207-215. [CrossRef] [PubMed]

4. Tsuchiya, M.; Sato, E.F.; Inoue, M.; Asada, A. Acupuncture enhances generation of nitric oxide and increases local circulation. Anesth. Analg. 2007, 104, 301-307. [CrossRef] [PubMed]

5. Li, X.; Li, Y.; Chen, J.; Zhou, D.; Liu, Y.; Li, Y.; Liu, J.; Guo, Y.; Guo, Y. The influence of skin microcirculation blood perfusion at zusanli acupoint by stimulating with lift-thrust reinforcing and reducing acupuncture manipulation methods on healthy adults. Evid.-Based Complement. Altern. Med. 2013. [CrossRef] [PubMed]

(C) 2018 by the authors. Licensee MDPI, Basel, Switzerland. This article is an open access article distributed under the terms and conditions of the Creative Commons Attribution (CC BY) license (http:/ / creativecommons.org/licenses/by/4.0/). 